\title{
Efeitos de doses de nitrogênio e potássio e densidade populacional sobre a classificação de bulbos de cebola
}

\author{
André May; Arthur B Cecílio Filho²; Diego R Queirós Porto²; Pablo F Vargas²; José Carlos Barbosa² \\ ${ }^{1}$ IAC, C. Postal 28, 13012-970, Campinas-SP; ${ }^{2}$ UNESP, FCAV, Via de acesso Prof. Paulo D. Castellane s/n, 14884-900 Jaboticabal-SP; \\ andre@tozan.com.br; rutra@fcav.unesp.br
}

\section{RESUMO}

O efeito de doses de nitrogênio e de potássio e da população de plantas foi avaliado na classificação de bulbos de duas cultivares de cebola. Os tratamentos foram arranjados em esquema fatorial $2 \times 4$ x 4 x 4, correspondentes a, respectivamente, cultivares (Optima e Superex), nitrogênio (0; 50; 100 e $150 \mathrm{~kg} \mathrm{ha}^{-1}$ de N), potássio (0; 75; 150 e $225 \mathrm{~kg} \mathrm{ha}^{-1}$ de $\mathrm{K}_{2} \mathrm{O}$ ) e população de plantas (60; 76; 92 e 108 plantas $\mathrm{m}^{-2}$ ). O delineamento experimental utilizado foi de blocos casualizados, com quatro repetições. Os fertilizantes utilizados foram nitrato de amônio e cloreto de potássio para o fornecimento de $\mathrm{N}$ e K, respectivamente. A aplicação de nitrogênio e de potássio reduziu para aproximadamente zero a porcentagem de bulbos nãocomerciais para as duas cultivares. A maior percentagem de bulbos com diâmetro transversal entre 50 e $70 \mathrm{~mm}$ foi alcançada com 60 plantas $\mathrm{m}^{-2}$ e com a aplicação de $150 \mathrm{~kg} \mathrm{ha}^{-1}$ de $\mathrm{N}$, principalmente para a Superex. As maiores porcentagens de bulbos duplos ocorreram na cultivar Superex, com a aplicação de $150 \mathrm{~kg} \mathrm{ha}^{-1}$ de N (17\% do total de bulbos produzidos) e sob população de 60 plantas $\mathrm{m}^{-2}$ (22\% do total de bulbos).

Palavras-chave: Allium cepa L., fertilização, população de plantas.

\begin{abstract}
Effect of onion bulb classification as a result of nitrogen and potassium levels and planting density

The effect of nitrogen and potassium fertilizer doses and planting densities were evaluated in bulb classification of two onion cultivars. The treatments were arranged in factorial $2 \times 4 \times 4 \times 4$, respectively correspondent by cultivars (Optima and Superex), nitrogen (0; 50; 150 and $150 \mathrm{~kg} \mathrm{ha}^{-1}$ of $\left.\mathrm{N}\right)$, potassium $\left(0 ; 75 ; 150\right.$ and $225 \mathrm{~kg} \mathrm{ha}^{-1}$ of $\mathrm{K}_{2} \mathrm{O}$ ) and planting density (60; 76; 92 and 108 plants $\mathrm{m}^{-2}$ ). The experimental design was of randomized blocks, with four replications The fertilizers ammonium nitrate and potassium chloride were employed to provide $\mathrm{N}$ and $\mathrm{K}$, respectively. The nitrogen and potassium application reduced to near to zero the incidence of noncomercial bulbs in both cultivars. The largest percentage of bulbs measuring 50 to $70 \mathrm{~mm}$ was reached with 60 plants $\mathrm{m}^{-2}$ and application of $150 \mathrm{~kg} \mathrm{ha}^{-1}$ of N, mainly for cv. Superex. Cultivar Superex presented the largest quantity of double bulbs, with the application of $150 \mathrm{~kg} \mathrm{ha}^{-1}$ of $\mathrm{N}$ (17\% of the total bulbs produced) and plant population of 60 plants $\mathrm{m}^{-2}$ (22\% of the total bulbs).
\end{abstract}

Keywords: Allium cepa L., fertilization, plant population.

\section{(Recebido para publicação em 22 de maio de 2006; aceito em 30 de agosto de 2007)}

$\mathrm{N}^{\mathrm{s}}$ o Brasil, a cebola é considerada a terceira hortaliça mais importante em valor econômico, sendo superada pela batata e pelo tomate. É consumida preferencialmente na forma in natura, em saladas, sendo também utilizada para temperos e condimentos. O consumo per capita brasileiro situa-se próximo de 4,7 $\mathrm{kg} \mathrm{ano}^{-1}$, enquanto na Argentina este valor é de $10,0 \mathrm{~kg}$ ano ${ }^{-1}$; no Uruguai, 7,7 $\mathrm{kg}^{\mathrm{ano}} \mathrm{-}^{-1}$ e no Paraguai, $7,0 \mathrm{~kg} \mathrm{ano}^{-1}$ (Boeing, 2002).

O maior produtor mundial de cebola é a China, com 601.000 ha e produtividade média de $20,7 \mathrm{t} \mathrm{ha} \mathrm{h}^{-1}$. A produtividade média no Brasil tem variado nos últimos anos, entre 15 e $17 \mathrm{t} \mathrm{ha}^{-1}$, em área cultivada de 62.000 ha, alcançando o décimo segundo lugar no ranking mundial em volume produzido (Boeing, 2002).

No Brasil, a cebola é plantada comercialmente desde a região Sul até o Nordeste. Em 2001, a produção nacio- nal foi de aproximadamente um milhão de $\mathrm{t}$, com a região Sul, representando $62,2 \%$ do volume comercializado, seguida pelo Sudeste, com $25,2 \%$ e pelo Nordeste, com 12,3\%. Os estados de Santa Catarina, São Paulo e Rio Grande do Sul são, atualmente, os maiores produtores nacionais (Resende et al., 2002).

Com base na necessidade de bulbos de tamanho médio (50 a 70 mm de diâmetro), os produtores rurais têm buscado no adensamento de plantas a possibilidade de obter cebolas com melhor uniformidade na classificação. A competição por água, luz e nutrientes faz com que o tamanho dos bulbos e a produtividade total variem conforme a população de plantas na área (Nichols, 1967). Assim, torna-se importante o estudo da interação entre os fatores doses de nutrientes e população de plantas.

Santos et al. (2000) estudaram a cv. Texas Grano em diferentes espaçamentos $(0,05 ; 0,10$ e 0,15 m entre plantas na li- nha), utilizando 0,3 m entre linhas. Observaram que a redução no espaçamento foi diretamente responsável pela redução na massa do bulbo, e, consequentemente, no aumento da proporção de bulbos com menor diâmetro.

Dellacecca \& Lovato (2000), estudando três densidades de semeio direto (26,6; 40 e 80 plantas $\mathrm{m}^{-2}$ de cebola), verificaram que sob a menor densidade de plantas ocorreu a maior massa do bulbo (189,0 g) e sob a maior densidade a menor massa (105,5 g). Os autores não observaram efeito da densidade de plantas sobre o formato do bulbo. Brewster (1994) cita que para a obtenção de bulbos de cebola graúdos, a população de plantas deve estar entre 25 e 50 plantas $\mathrm{m}^{-2}$, e para a obtenção de bulbos entre 50 e 70 mm de diâmetro transversal, entre 50 e 100 plantas $\mathrm{m}^{-2}$.

Por participar da constituição de proteínas, o nitrogênio contribui marcadamente para a melhoria da pro- 
dução de cebola. Esse nutriente é absorvido em grande quantidade, superado apenas pelo potássio (Vidigal et al., 2000). No entanto, há grande variação na absorção do nutriente, em função de fatores como cultivar, densidade populacional e atributos do solo.

Aujla \& Madan (1992) obtiveram as melhores produtividades de cebola com a aplicação de $100 \mathrm{~kg} \mathrm{ha}^{-1}$ de $\mathrm{N}$, durante dois anos de estudo, com doses variando de 0 a $150 \mathrm{~kg} \mathrm{ha}^{-1}$. Para Henriksen (1987), a melhor dose de $\mathrm{N}$ aplicada na forma de nitrato de cálcio foi $120 \mathrm{~kg} \mathrm{ha}^{-1}$ de $\mathrm{N}$ (variando de 0 a $180 \mathrm{~kg} \mathrm{ha}^{-1}$ ) com produtividade de $61,5 \mathrm{t} \mathrm{ha}^{-1}$ de bulbos comerciais e população de 80 plantas $\mathrm{m}^{-2}$, cultivadas no sistema de transplante de mudas. Porwal \& Singh (1993) conseguiram as melhores produtividades com $50 \mathrm{~kg} \mathrm{ha}^{-1}$ de $\mathrm{N}$, enquanto em maior dose (150 kg ha ${ }^{-1}$ de $\mathrm{N}$ ), as produções foram menores. Da mesma forma que Rana \& Sharma (1993), altas doses de N (120 $\mathrm{kg} \mathrm{ha}^{-1}$ ) não influenciaram significativamente na produção de bulbos de cebola, havendo efeito positivo de doses até $80 \mathrm{~kg} \mathrm{ha}^{-1}$ de N. (Vidigal et al., 2000) cita que algumas zonas de cultivo de Minas Gerais chegam a ser fertilizadas com $175 \mathrm{~kg} \mathrm{ha}^{-1}$ para a cultivar Alfa Tropical, mas com baixa produtividade (20 $\mathrm{t} \mathrm{ha}^{-1}$ ), em razão da baixa tecnologia de cultivo empregada.

Brewster (1994) relata que a maioria das recomendações de aplicação de $\mathrm{K}$ em cebola sugerem valores entre 50 e $250 \mathrm{~kg} \mathrm{ha}^{-1}$, apresentando resultados bastante variáveis em função da fertilidade do solo. Esse nutriente é reportado por Marschner (1995) como importante para espécies que armazenam reservas em órgãos como o bulbo, principalmente, relacionando-o com o tamanho deles.

O presente trabalho teve como objetivo avaliar o efeito de doses de $\mathrm{N}$ e K sobre a classificação de bulbos em cultura de cebola estabelecida por semeadura direta, em quatro densidades populacionais.

\section{MATERIAL E MÉTODOS}

O experimento foi conduzido no município de São José do Rio Pardo, SP, com altitude de aproximadamente 900 m. O clima da região é do tipo tropical, com inverno seco, classificado como Aw segundo Köppen, descrito por Vianello \& Alves (1991). A precipitação pluvial média anual situa-se em torno de 1.400 $\mathrm{mm}$, e as temperaturas médias anuais máxima e mínima são de $31,6^{\circ} \mathrm{C}$ e $17,9^{\circ} \mathrm{C}$, respectivamente.

O delineamento experimental utilizado foi de blocos ao acaso, com os tratamentos em esquema fatorial 2 × 4 × 4 x 4, com quatro repetições. Foram avaliados duas cultivares (Híbridos Optima e Superex); quatro populações de plantas $\left(60,76\right.$, 92 e 108 plantas $\left.\mathrm{m}^{-2}\right)$; quatro doses de nitrogênio $(0,50,100$ e 150 $\mathrm{kg} \mathrm{ha}^{-1}$ de $\mathrm{N}$ ), na forma de nitrato de amônio, e quatro doses de potássio ( 0 , 75, 150 e $225 \mathrm{~kg} \mathrm{ha}^{-1}$ de $\mathrm{K}_{2} \mathrm{O}$ ), na forma de cloreto de potássio. As parcelas foram localizadas em canteiros de 1,2 m de largura, com cinco linhas longitudinais ao canteiro e com comprimento de 2,5 m. O espaçamento entre linhas foi de $0,275 \mathrm{~m}$.

O solo da área do experimento é classificado como Latossolo Vermelho Amarelo de textura argilosa (Embrapa, 1999). A análise da amostra do solo, retirada da profundidade de 0 a $0,2 \mathrm{~m}$, feita em laboratório resultou: $\mathrm{pH}$ em $\mathrm{CaCl}_{2}=$ 5,2; $\mathrm{MO}=25 \mathrm{~g} \mathrm{~kg}^{-1}$ e P-resina= $24 \mathrm{mg}$ $\mathrm{dm}^{-3}$. Os teores de $\mathrm{K}, \mathrm{Ca}, \mathrm{Mg}, \mathrm{H}+\mathrm{Al}$ foram, respectivamente, 1,4; 31; 10 e 28 $\mathrm{mmol}_{\mathrm{c}} \mathrm{dm}^{-3}$ e a CTC igual a 70,4 mmol $\mathrm{dm}^{-3}$.

Com base na análise do solo e nas recomendações de Trani et al. (1996) para a calagem e adubação da cultura da cebola, sistema de transplantio, fezse aplicação de calcário, um mês antes da semeadura, para elevar a saturação por bases a 70\%. Aplicaram-se, na semana da instalação do experimento: 300 $\mathrm{kg} \mathrm{ha}^{-1}$ de $\mathrm{P}_{2} \mathrm{O}_{5}$, como superfosfato simples, à lanço e em área total, e 1,5 kg ha-1 de B, como bórax.

Dois dias antes da semeadura, foram aplicados $30 \mathrm{~kg} \mathrm{ha}^{-1}$ de $\mathrm{N}$ e $40 \mathrm{~kg} \mathrm{ha}^{-1}$ de $\mathrm{K}_{2} \mathrm{O}$ em todos os tratamentos, exceto na testemunha. A incorporação foi feita com enxada, seguida de irrigação com lâmina de $10 \mathrm{~mm}$. O restante do nitrogênio e potássio de cada tratamento foi parcelado em quantidades iguais e aplicado aos 30, 45 e 60 dias após a semeadura (DAS). A semeadura foi realizada em 07/03/04 em covas de $3 \mathrm{~cm}$ de profundidade. Em cada cova foram colocadas cinco a sete sementes de cebola, sendo realizado desbaste após 15 dias para estabelecer o devido espaçamento entre plantas.

A aplicação de herbicidas, fungicidas e inseticidas foi feita de acordo com a necessidade da cultura. As irrigações, utilizando o sistema de aspersão convencional foram diárias durante as primeiras semanas após a semeadura e a cada quatro dias em complementação às chuvas. A lâmina média de irrigação aplicada foi de $10 \mathrm{~mm}$.

O ponto de colheita (data) foi determinado quando $70 \%$ das plantas "estalaram”, caracterizado pelo amolecimento do pseudocaule e tombamento da parte aérea, baseado na proposta de Finger \& Casali (2002). A colheita foi iniciada 140 dias após a semeadura. As plantas foram arrancadas e deixadas sobre os canteiros com as folhas distribuídas sobre os bulbos para que ficassem protegidos da radiação direta. Após quatro dias de insolação e após a eliminação da parte aérea e das raízes, os bulbos foram retirados do local e encaminhados para o laboratório onde foram feitas as avaliações para classificação.

A estimativa da produtividade ( $\mathrm{t} \mathrm{ha} \mathrm{h}^{-1}$ ) foi realizada a partir da pesagem dos bulbos colhidos das três linhas centrais da parcela (deixando-se uma linha de bordadura de cada lado) em um metro linear de canteiro e feita a proporção para 1 ha com $5.900 \mathrm{~m}^{2}$ efetivamente cultivados, descontando os espaços livres entre os canteiros.

Os bulbos foram classificados pelo maior diâmetro transversal, adaptando a classificação da CEAGESP (2001), em Classe 0 ou refugo $=<15 \mathrm{~mm}$; Classe $1=$ 15 a $35 \mathrm{~mm}$; Classe 2= 35 a $50 \mathrm{~mm}$; Classe $3=50$ a $70 \mathrm{~mm}$ e Classe $4=70$ a $90 \mathrm{~mm}$, com os resultados expressos em porcentagem da produção total. Anotouse também a quantidade de bulbos duplos considerados com defeito leve.

Os dados foram submetidos à análise de variância (teste F) pelo programa estatístico Estat (UNESP, FCAV, Campus Jaboticabal) e transformados em arcseno $\{$ raiz $[(x+a l f a) / 100)\}$, visando distribuição normal dos valores. Os dados em função das variáveis quanti- 
Tabela 1. Análise da superfície de resposta para a característica Classe 0 (\%) em função das doses de N e da população de plantas estudada para as cultivares de cebola Optima e Superex e para a característica Classe 1 (\%) em função das doses de N e K para as cultivares Optima e Superex (analysis of response for the characteristics Class 0 (\%) as a result of $\mathrm{N}$ doses and of planting desity for onion cultivars Optima and Superex and, for the charactesistic Class 1 (\%) as a result of $\mathrm{N}$ and K doses for the cultivars Optima and Superex). Jaboticabal, UNESP, 2006.

\begin{tabular}{|c|c|c|c|c|c|}
\hline \multirow{2}{*}{ Parâmetros do modelo } & \multicolumn{2}{|c|}{ Classe $0(\%)$} & \multirow{2}{*}{ Parâmetros do modelo } & \multicolumn{2}{|c|}{ Classe $1(\%)$} \\
\hline & Optima & Superex & & Optima & Superex \\
\hline$\overline{b_{0} \text { (Intercepto) }}$ & $-53,140692$ & 8,090723 & $\mathrm{~b}_{0}$ (Intercepto) & 54,619450 & 59,833150 \\
\hline $\mathrm{b}_{1}(\mathrm{~N})$ & $-0,094737$ & $-0,246730$ & $b_{1}(N)$ & $-0,324036$ & $-0,429582$ \\
\hline$b_{2}(P P)$ & 1,510859 & 0,206407 & $\mathrm{~b}_{2}(\mathrm{~K})$ & $-0,094527$ & $-0,040778$ \\
\hline$b_{3}\left(N^{2}\right)$ & 0,000982 & 0,000826 & $b_{3}(\mathrm{~N} 2)$ & 0,000946 & 0,001185 \\
\hline $\mathrm{b}_{4}(\mathrm{~N} \times \mathrm{PP})$ & $-0,002352$ & $-0,000196$ & $\mathrm{~b}_{4}(\mathrm{~N} \times \mathrm{K})$ & 0,000436 & 0,000001173 \\
\hline $\mathrm{b}_{5}\left(\mathrm{PP}^{2}\right)$ & $-0,006658$ & $-0,000403$ & $\mathrm{~b}_{5}\left(\mathrm{~K}^{2}\right)$ & 0,000170 & 0,000084667 \\
\hline Teste F para o modelo & $18,241^{* *}$ & $6,473^{* *}$ & Teste F para o modelo & $16,119^{* *}$ & $20,353^{* *}$ \\
\hline $\mathrm{R}^{2}$ & 0,90 & 0,76 & $\mathrm{R}^{2}$ & 0,89 & 0,91 \\
\hline CV $(\%)$ & 37,81 & 65,26 & CV $(\%)$ & 10,64 & 16,39 \\
\hline Ponto crítico $\mathrm{N}$ & 151,9514 & 174,6761 & Ponto crítico $\mathrm{N}$ & 152,2691 & 181,1396 \\
\hline Ponto crítico PP & 86,6291 & 213,6685 & Ponto crítico $\mathrm{K}$ & 82,4001 & 239,5598 \\
\hline Tipo & Ponto de sela & Ponto de sela & Tipo & Mínimo & Mínimo \\
\hline Valor predito no ponto crítico & 5,1038 & 8,5931 & Valor predito no ponto crítico & 26,0538 & 16,0416 \\
\hline
\end{tabular}

**Significativo a $1 \%$ de probabilidade, pelo Teste $\mathrm{F}$ (**significant at $1 \%$ probability, $\mathrm{F}$ test).

tativas foram submetidos à análise de regressão, sem transformação. Os modelos de regressão (linear, quadrático ou cúbico) foram escolhidos pelo maior coeficiente de determinação $\left(\mathrm{R}^{2}\right)$ que tenha apresentado maior significância pelo teste F. Foram utilizados os programas, SAS, para as análises estatísticas dos dados originais e programa Statistica, para ajuste das equações polinomiais múltiplas.

\section{RESULTADOS E DISCUSSÃO}

As máximas produtividades obtidas, 72,02 t ha-1 para a 'Optima' e 78,91 t ha-1 para a 'Superex', foram superiores àquelas tradicionalmente obtidas pelos produtores do Brasil (média nacional 15 a $17 \mathrm{t} \mathrm{ha}^{-1}$ de bulbos) (Boeing, 2002).

As cultivares Optima e Superex produziram 13,15 e 10,75\% dos bulbos distribuídos na Classe 0 (refugo), respectivamente. Em quase a totalidade dos casos, a presença de grandes quantidades de bulbos dessa classificação é descontada no valor total pago ao produtor.

Constatou-se significância da interação entre doses de nitrogênio e população de plantas sobre a classificação dos bulbos. As menores percentagens de Classe 0 (refugo) foram obtidas sob menores populações de plantas combinadas às altas doses de nitrogênio. As maiores percentagens de bulbos Classe 0 foram observadas na cultivar Optima (29,4\%), com valor $26,6 \%$ superior à 'Superex' (23,2\%) para a máxima produção de bulbos nessa categoria (Tabela 1). Isto foi observado, principalmente na cultivar Optima pois, quando se fez a aplicação de altas doses de N (150 kg ha-1), a variação na porcentagem de bulbos na Classe 0 foi pequena mediante a alteração na população de plantas. Por outro lado, essa diferença foi grande quando se reduziu a dose de $\mathrm{N}$ aplicada.

As menores percentagens de bulbos Classe 0 (2,9\%) foram obtidas, para a cultivar Optima, com aplicação de 115 $\mathrm{kg} \mathrm{ha}^{-1}$ de $\mathrm{N}$ e 108 plantas $\mathrm{m}^{-2}$ até $150 \mathrm{~kg}$ $h^{-1}$ de $\mathrm{N}$ e $103 \mathrm{pl} \mathrm{m}^{-2}$, ou entre as doses de 50 e $150 \mathrm{~kg} \mathrm{ha}^{-1}$ de $\mathrm{N}$ combinadas com população de 60 a 70 plantas $\mathrm{m}^{-2}$. Já para a cultivar Superex, os menores valores $(1,3 \%)$ foram alcançados para doses de $\mathrm{N}$ superiores a $85 \mathrm{~kg} \mathrm{ha}^{-1}$, combinadas com população de 60 e 80 plantas $\mathrm{m}^{-2}$ (Tabela 1$)$.

A Classe 1 foi significativamente influenciada pela interação entre cultivares e doses de nitrogênio e doses de nitrogênio e potássio. Assim como observado para a Classe 0 , houve aumento da percentagem de bulbos classificados como Classe 1 à medida que aumentou a população de plantas ( $\widehat{y}=11,785$ $\left.+0,2751 \mathrm{x}, \mathrm{R}^{2}=0,77^{* *}\right)$. Sob a maior população de plantas (108 plantas $\mathrm{m}^{-2}$ ) houve $42 \%$ dos bulbos na Classe 1 , sendo $33 \%$ superior ao obtido sob população de 60 plantas $\mathrm{m}^{-2}$, demonstrando, mais uma vez, que a competição entre as plantas proporcionou produção de bulbos com menores diâmetros.

Variando a dose de $\mathrm{K}_{2} \mathrm{O}$ de 0 para $225 \mathrm{~kg} \mathrm{ha}^{-1}$, em qualquer dose de $\mathrm{N}$, a variação na percentagem da Classe 1 foi pequena. Para as duas cultivares, a aplicação de doses de $\mathrm{N}$ acima de $100 \mathrm{~kg}$ ha $^{-1}$ reduziu a distribuição de bulbos na Classe 1 para valores inferiores a 30\%.

Para a Classe 2, houve interação significativa entre os fatores cultivares e doses de nitrogênio, doses de nitrogênio e doses de potássio e para doses de nitrogênio e população de plantas. Para obter as menores percentagens distribuídas na Classe 2, faz-se necessário reduzir a dose de nitrogênio associado à elevação na população de plantas.

A aplicação de potássio reduziu significativamente a quantidade de bulbos na Classe 0 até a dose de $150 \mathrm{~kg} \mathrm{ha}^{-1}$ de $\mathrm{K}_{2} \mathrm{O}$, com pequeno crescimento entre essa dose e $225 \mathrm{~kg} \mathrm{ha}^{-1}$ de $\mathrm{K}_{2} \mathrm{O}(\hat{\mathrm{y}}=13,572$ $\left.0,0706 \mathrm{x}+0,0002 \mathrm{x}^{2}, \mathrm{R}^{2}=0,99 * *\right)$.

O potássio influenciou pouco na expressão da Classe 2, ou seja, para incrementar o percentual de classificação da produção na Classe 2 foi necessária aplicação de altas doses do potássio, 
Tabela 2. Análise da superfície de resposta para as características Classe 2 (\%) em função das doses de N e K para as populações de plantas de 60 plantas $\mathrm{m}^{-2}$ (1); 76 plantas $\mathrm{m}^{-2}$ (2); 92 plantas $\mathrm{m}^{-2}$ (3) e 108 plantas $\mathrm{m}^{-2}$ (4), para a cultivar Optima e Classe 2 (\%) em função das doses de N e K para populações de 60 plantas $\mathrm{m}^{-2}$ (5); 76 plantas $\mathrm{m}^{-2}$ (6); 92 plantas $\mathrm{m}^{-2}$ (7) e 108 plantas $\mathrm{m}^{-2}$ (8) para a cultivar Superex e Classe 2 (\%) em função das doses de $\mathrm{N}$ e da população de planta estudada para as cultivares Optima (9) e Superex (10), respectivamente (analysis of response for the characteristics Class 2 (\%) as a result of $\mathrm{N}$ and $\mathrm{K}$ doses for populations of 60 plants $\mathrm{m}^{-2}$ (1); 76 plants $\mathrm{m}^{-2}$ (2); 92 plants $\mathrm{m}^{-2}$ (3) and 108 plants $\mathrm{m}^{-2}$ (4), for the cultivar Optima and, Class 2 (\%) as a result of $\mathrm{N}$ and $\mathrm{K}$ doses for populations of 60 plants $\mathrm{m}^{-2}$ (5); 76 plants $\mathrm{m}^{-2}$ (6); 92 plants $\mathrm{m}^{-2}$ (7) and 108 plants $\mathrm{m}^{-2}$ (8) for cultivar Superex and, Class 2 (\%) as a result of $\mathrm{N}$ doses and of plant populations for cultivars Optima (9) and Superex (10), respectively). Jaboticabal, UNESP-FCAV, 2006.

\begin{tabular}{|c|c|c|c|c|c|c|c|c|c|c|}
\hline $\begin{array}{l}\text { Parâmetros } \\
\text { do modelo }\end{array}$ & 1 & 2 & 3 & 4 & 5 & 6 & 7 & 8 & 9 & 10 \\
\hline$b_{0}$ (Intercepto) & 25,646950 & 16,6751 & 15,378050 & 11,533050 & 12,4819 & 18,534300 & 12,262800 & 4,189950 & $-96,382378$ & 8,090723 \\
\hline$b_{1}(N)$ & 0,211218 & 0,185685 & 0,254659 & 0,353128 & 0,3513 & 0,226563 & 0,467003 & 0,405545 & $-0,199968$ & $-0,246730$ \\
\hline$b_{2}(K)$ & 0,081376 & 0,046270 & 0,163804 & 0,039833 & 0,0893 & 0,049947 & 0,0053399 & 0,110149 & 2,997333 & 0,206407 \\
\hline$b_{3}\left(N^{2}\right)$ & $-0,000858$ & $-0,000358$ & $-0,000766$ & $-0,001014$ & $-0,001271$ & $-0,000608$ & $-0,001943$ & $-0,000918$ & $-0,000356$ & 0,000826 \\
\hline $\mathrm{b}_{4}(\mathrm{~N} \times \mathrm{K})$ & $-0,000434$ & $-0,000183$ & $-0,000164$ & $-0,000206$ & $-0,000595$ & $-0,000129$ & 0,000343 & $-0,000354$ & 0,003106 & $-0,000196$ \\
\hline $\mathrm{b}_{5}\left(\mathrm{~K}^{2}\right)$ & $-0,000275$ & 0,000146 & $-0,000543$ & $-0,00002666$ & 0,000006152 & $-0,00005078$ & $-0,000229$ & $-0,000296$ & $-0,016665$ & $-0,000403$ \\
\hline $\begin{array}{l}\text { Teste F para o } \\
\text { modelo }\end{array}$ & 1,644 ns & $6,257^{* *}$ & $3,287^{*}$ & $7,609 * *$ & $2,503 \mathrm{~ns}$ & $4,619^{* *}$ & $17,582^{* *}$ & $6,773^{* *}$ & $28,192^{* *}$ & $6,473^{* *}$ \\
\hline $\mathrm{R}^{2}$ & 0,45 & 0,76 & 0,63 & 0,79 & 0,55 & 0,70 & 0,90 & 0,77 & 0,93 & 0,76 \\
\hline CV (\%) & 20,85 & 18,09 & 23,3027 & 21,16 & 26,15 & 18,18 & 16,22 & 30,14 & 10,39 & 65,26 \\
\hline Ponto crítico N & 107,0571 & 258,2725 & 152,5923 & 161,6736 & 149,0700 & 155,1825 & 139,6264 & 209,0905 & 188,3211 & 174,6761 \\
\hline Ponto crítico K & 63,5330 & 3,2260 & 127,7376 & 122,5057 & $-46,5359$ & 295,3217 & 220,8337 & 61,1567 & 107,4808 & 213,6685 \\
\hline Tipo & Máximo & $\begin{array}{l}\text { Ponto de } \\
\text { sela }\end{array}$ & Máximo & Máximo & $\begin{array}{l}\text { Ponto de } \\
\text { sela }\end{array}$ & Máximo & Máximo & Máximo & Máximo & $\begin{array}{l}\text { Ponto de } \\
\text { sela }\end{array}$ \\
\hline $\begin{array}{l}\text { Valor predito } \\
\text { no ponto } \\
\text { crítico }\end{array}$ & 39,5330 & 40,7284 & 45,2695 & 42,5187 & 36,5913 & 43,4889 & 50,7619 & 49,9559 & 45,8665 & 8,5931 \\
\hline
\end{tabular}

**Significativo a $1 \%$ de probabilidade, pelo Teste $\mathrm{F}$ (**significant at $1 \%$ probability, $\mathrm{F}$ test).

para mesma dose de nitrogênio (Tabela 2). As máximas percentagens de bulbos na Classe 2 sob populações de 60; 76; 92 e 108 plantas $\mathrm{m}^{-2}$, foram 38; 47; 43 e 40\%, para a 'Optima' e 40; 41; 47 e 45\%, para a 'Superex'. A grande quantidade de bulbos com diâmetro entre 35 e 50 mm não é interessante uma vez que o mercado prefere bulbos com diâmetro entre 50 e 70 mm. Verifica-se a existência de diferença entre as cultivares para a população de plantas, que maximizou a porcentagem de bulbos na Classe 2. A cultivar Optima teve 47\% da produção na Classe 2 com menor densidade de plantas (76 plantas $\mathrm{m}^{-2}$ ) em relação à 'Superex' (92 plantas $\mathrm{m}^{-2}$ ).

Para a percentagem de bulbos na Classe 3, houve efeito significativo dos fatores doses de potássio e população de plantas isoladamente, e interação significativa dos fatores cultivares e doses de nitrogênio.

Embora tenha havido efeito significativo do k na expressão da característica Classe 3 , as médias observadas $(17,0 ; 23,4 ; 20,3$ e $22,6 \%$ de bulbos na Classe 3) não se ajustaram à equação de regressão polinomial.

Constatou-se aumento de $60 \%$ na classificação de bulbos Classe 3, com a redução da população de 108 para 60 plantas $\mathrm{m}^{-2}\left(\hat{\mathrm{y}}=51,717-0,3678 \mathrm{x} ; \mathrm{R}^{2}=\right.$ $\left.0,98^{* *}\right)$. Considerando que os bulbos preferidos pelo mercado consumidor são os classificados como Classe 3 (Silva et al., 1991), para que se obtenha maiores percentagens nessa classe, deve-se usar menor população, uma vez que a elevada população de plantas reduz o tamanho de bulbos, em razão da maior competição entre as plantas.

Maier et al. (1990) também observaram que o nitrogênio foi muito importante para o aumento da quantidade produzida de bulbos comerciais. Kanton et al. (2002) citam que a população de plantas é o fator mais importante para a produção de bulbos com diâmetros comercialmente mais desejáveis.

A fertilização nitrogenada elevou a quantidade de bulbos distribuídos na Classe 3. Até $92 \mathrm{~kg} \mathrm{ha}^{-1}$ de $\mathrm{N}$, que representa o ponto de cruzamento das duas linhas de tendência traçadas para cada cultivar estudada, não houve diferenças expressivas entre as cultivares (Superex: $\widehat{y}=2,8161+0,3219 x-0,0006 x^{2}$;
$\mathrm{R}^{2}=0,99 * *$ e Optima: $\hat{\mathrm{y}}=4,5505+$ $\left.0,3545 \mathrm{x}-0,0013 \mathrm{x}^{2} ; \mathrm{R}^{2}=0,98 * *\right)$. Para a Optima houve, quando da aplicação de 100 a $150 \mathrm{~kg} \mathrm{ha}^{-1}$ de $\mathrm{N}$, tendência de queda na produção de bulbos nesta classificação. Já para 'Superex', houve incremento de 32,8\% no percentual de bulbos na Classe 3, quando a dose de $\mathrm{N}$ passou de 100 para $150 \mathrm{~kg} \mathrm{ha}^{-1}$.

Asiegbu (1989), estudando o efeito da aplicação de 0; 100; 200 e 300 kg ha${ }^{1}$ de $\mathrm{N}$ sobre cebolas transplantadas com população de 500.000 plantas ha ${ }^{-1}$, verificou maiores proporções de bulbos entre os diâmetros 45 e 64 mm para a aplicação de $100 \mathrm{~kg} \mathrm{ha}^{-1}$ de $\mathrm{N}$, representando cerca de $40 \%$ do total de bulbos produzidos. Sob doses mais elevadas de $\mathrm{N}$ não houve aumento significativo da proporção de bulbos produzidos nesta classificação.

Para a Classe 4 (70 a 90 mm), houve interação significativa apenas para os fatores doses de nitrogênio e população de plantas, não havendo efeitos significativos para os demais fatores isoladamente.

A percentagem de bulbos na Classe 4 para as populações entre 76 e 108 plan- 
tas $\mathrm{m}^{-2}$ foi inferior a $0,65 \%$ do total de bulbos colhidos, cujo valor foi maximizado no estande de 76 plantas $\mathrm{m}^{-2}$ e aplicação de $150 \mathrm{~kg} \mathrm{ha}^{-1}$ de $\mathrm{N}(\widehat{\mathrm{y}}=$ $\left.-0,0508+0,0047 x, R^{2}=0,75^{*}\right)$. Na menor população de plantas avaliada (60 plantas $\mathrm{m}^{-2}$ ), observou-se resposta quadrática da percentagem de bulbos na Classe 4 com o aumento do $\mathrm{N}$ aplicado $\left(\hat{y}=0,2207+0,0357 x-0,0001 x^{2} ; R^{2}=\right.$ $\left.0,81^{*}\right)$. Sob essa população de plantas, o percentual de bulbos na Classe 4 , que era $0,22 \%$, atingiu 3,3\% com $150 \mathrm{~kg}$ ha $^{-1}$ de N. O mercado consumidor não aceita grandes volumes de bulbos com os diâmetros da Classe 4, por isso, o sistema produtivo deve ser manejado de tal maneira a não se obter bulbos dessa classe.

Para bulbo duplo, houve interações significativas entre os fatores cultivares e doses de nitrogênio e cultivares e população de plantas. Os coeficientes de variação foram elevados (79,53\%) em razão do tipo de distribuição dessa característica.

Houve aumento da percentagem de bulbos duplos com o aumento da dose de potássio aplicada, conferindo grande efeito sobre a qualidade visual dos mesmos, passando de 5,3 para $7,22 \%$ de bulbos duplos com a aplicação de 0 e $225 \mathrm{~kg} \mathrm{ha}^{-1}$ de $\mathrm{K}_{2} \mathrm{O}(\hat{\mathrm{y}}=5,3332+$ $\left.0,0078 x ; R^{2}=0,54^{*}\right)$. Esse comportamento, provavelmente ocorreu porque, segundo Marschner (1995), como o potássio favorece a extensão celular nas plantas, a aplicação de altas doses promove o aparecimento de maior quantidade de bulbo duplo.

A 'Optima', mesmo sob as maiores doses de $\mathrm{N}$, não teve aumento na quantidade produzida de bulbos duplos, ocorrendo o oposto para a 'Superex', que, mesmo sem a aplicação de N, produziu $5,6 \%$ de bulbos duplos $(\hat{y}=5,6595+$ $\left.0,0779 x ; R^{2}=0,98^{* *}\right)$. As causas para o surgimento de centros duplos em bulbo de cebola são diversas; por exemplo, excesso de fertilização nitrogenada. Hassan (1984) relata que com a aplicação de doses acima de $90 \mathrm{~kg} \mathrm{ha}^{-1}$ de $\mathrm{N}$, com produtividades ao redor de 12,94 t $\mathrm{ha}^{-1}$, houve aumento da quantidade dos bulbos duplos, passando de 12,49 para 21,47\%. Apesar disso, o genótipo é o que mais influencia.

A presença de grande quantidade de bulbos duplos no total de bulbos produzidos para a 'Superex' (média cerca de 17\%) pode influenciar na redução dos preços pagos pelo produto, uma vez que essa característica é considerada um defeito fisiológico que dificulta a comercialização (CEAGESP, 2001).

A população de plantas também influenciou o aparecimento de bulbo duplo para a cultivar Superex, sendo que, quanto menor o número de plantas por área, maior a percentagem de bulbos duplos, chegando até cerca de $20 \%$ no total de bulbos produzidos sob população de 60 plantas $\mathrm{m}^{-2}$, reduzindo para 2,9\% sob a maior população estudada $\left(\widehat{y}=41,636-0,3587 x ; R^{2}=0,96 * *\right)$. Para a 'Optima', foi verificada também tendência de redução na percentagem de bulbos duplos quando houve elevação no número de plantas por área $(\hat{y}=$ $\left.4,1832-0,0374 x+, \mathrm{R}^{2}=0,86 * *\right)$, porém, de forma menos expressiva que para a Superex. Sob menor população de plantas, condição mais favorável à expressão da anormalidade, a percentagem de bulbos duplos, para a 'Optima', foi cerca de 1,9\%; bem abaixo dos valores obtidos para a outra cultivar. Supõe-se que a menor porcentagem de bulbos duplos sob maiores populações de plantas em ambas as cultivares decorre da maior dificuldade de evolução do centro duplo no interior dos bulbos nessas condições de maior adensamento, em razão da maior competição entre as plantas e crescimento do bulbo. Desse modo, o que efetivamente contribuiu para a menor ocorrência de bulbos duplos foi a população de plantas, uma vez que para a 'Optima', quando cultivada sob densidade de 108 plantas $\mathrm{m}^{-2}$, a quantidade produzida foi praticamente zero, e para a 'Superex', $2,8 \%$.

Assim, pode-se concluir que as aplicações de nitrogênio e potássio, combinadas com alterações na população de plantas, reduziram para praticamente zero a porcentagem de bulbos não-comerciais para as duas cultivares. A maior percentagem de bulbo com diâmetro transversal entre 50 e $70 \mathrm{~mm}$ foi alcançada com 60 plantas $\mathrm{m}^{-2}$ e com a aplicação de $150 \mathrm{~kg} \mathrm{ha}^{-1}$ de N, princi- palmente para a Superex, embora resultando na maior porcentagem de bulbos duplos para essa cultivar.

\section{AGRADECIMENTOS}

Os autores agradecem à FAPESP, pelo auxílio à pesquisa concedido; ao CNPq pela bolsa de doutorado; às empresas Agristar do Brasil LTDA e Takii do Brasil LTDA, nas pessoas de Maurício Coutinho Pellegrini e Eduardo Hideomi Seo, respectivamente e à Rosemari Teotônio Rodrigues e à Salete Anelise Nappi, pela ajuda nos trabalhos de campo.

\section{REFERÊNCIAS}

ASIEGBU JE. 1989. Response of onion to lime and fertilizer $\mathrm{N}$ in a tropical Ultisol. Tropical Agriculturae 66: 161-166.

AUJLA TS; MADAN PS. 1992. Response of onion (Allium cepa L.) to irrigation, nitrogen fertilizer and row spacing on deep sandy-loam soil in subtropical monsoon region. Indian Journal of Agricultural Sciences 62: 129-34.

BOEING G. 2002. Fatores que afetam a qualidade da cebola na agricultura familiar catarinense. Florianópolis: Instituto CEPA/SC. 88 p.

BREWSTER JL. 1994. Onion and other vegetable Alliums. Wellesbourne: Horticulture Research International/CAB Internacional. $236 \mathrm{p}$.

COMPANHIA DE ENTREPOSTOS E ARMAZÉNS GERAIS DO ESTADO DE SÃO PAULO. CEAGESP. 2001. Programa Brasileiro para a melhoria dos padrões comerciais $e$ embalagens de hortigranjeiros. São Paulo.

DELLACECCA V; LOVATO AFS. 2000. Effects of different plant densities and planting systems on onion (Allium cepa L.) bulb quality and yield. Acta Horticulturae 533: 197-201.

EMBRAPA. 1999. Sistema Brasileiro de Classificação de Solos. Rio de Janeiro: Embrapa Solos. 412p.

FINGER FL; CASALI VWD. 2002. Colheita, cura e armazenamento da cebola. Informe Agropecuário 23:93-98.

HASSAN MS. 1984. Effects of frequency of irrigattion and fertilizer nitrogen on yield and quality of onions (Allium cepa L.) in the arid tropics of Sudan. Acta Horticulturae 8: 341-346.

HENRIKSEN K. 1987. Effect of $\mathrm{N}$ and $\mathrm{P}$ fertilization on yield and harvest time in bulb onions. Acta Horticulturae 198: 207-215.

KANTON RAL; ABBEY L; HILLA RG; TABIL MA; JAN ND. 2002. Density affects plant development and yield of bulb onion (Allium сера L.) in northern Ghana. Journal of Vegetable Crop Production 8: 15-25.

MAIER NA; DAHLENBURG AP; TWIGDEN TK. 1990. Effect of nitrogen on the yield and quality of irrigated onions (Allium cepa L.) cv. Cream Gold grown on siliceous sands. Australian Journal of Experimental Agriculture 30: 845-851. 
MARSCHNER H. 1995. Mineral nutrition of higher plants. New York: Academic Press. 889 p.

NICHOLS MA. 1967. A note on a plant density and fertilizer experiment with onions in New Zealand. Horticultural Research 7: 144-7.

PORWAL WK; SINGH MM. 1993. Effect of nitrogen and weed management on onion. Indian Journal of Agronomy 38: 74-77.

RANADS; SHARMARP. 1993. Growth analysis and bulbs yield of onion as affected by irrigation and nitrogen. Indian Journal of Agronomy: 38: 676-677.

RESENDE LMA; MASCARENHAS MHT; SIMÃO MLR. 2002. Panorama da produção e da comercialização da cebola em Minas Gerais. Informe Agropecuário 23: 7-19.
SANTOS HS; TANAKA MT; WATANABE SH; ARANTES PAZ; IVONE TT. 2000. Produção de cebola em função de tamanho de muda e espaçamento. Horticultura Brasileira 18: 556557

SILVA E; TEIXEIRA LAJ; AMADO TJC. 1991. The increase in onion production in Santa Catarina State, South Brazil. Onion Newsletter for the Tropics 3: 7-9.

TRANI PE; TAVARES M; SIQUEIRA WJ. 1996. Cebola (sistema de mudas). In: RAIJ, B. Van; CANTARELLA, H.; QUAGGIO, J. A.; FURLANI, A. M. C. Recomendações de adubação e calagem para o estado de São Paulo, Campinas-SP: IAC. p. 176. (Boletim Técnico 100).
VIANELLO RL; ALVES AR. 1991. Meteorologia básica e aplicações. Viçosa, UFV. Imprensa Universitária. 449 p.

VIDIGAL SM; PEREIRA PRG; SEDIYAMACS; SEDIYAMA MAN; FONTES PCR. 2000 Produção de cebola influenciada por doses, fontes e parcelamento de nitrogênio em diferentes épocas de cultivo no verão. Horticultura Brasileira 18: 814-815 